\title{
Arectge Ontemal
}

\section{PESO DO ÚtERO APÓS LIGADURA NO RAMO OVARIANO dO VASO UTERINO}

Roberto Adelino de Almeida Prado*, tsutomu Aoki, José Mendes Aldrigh

Trabalho realizado no Departamento de Ginecologia e Obstetrícia da Faculdade de Medicina da Santa Casa de São Paulo, SP

*Correspondência:

Rua Dr. Cesário Mota

Júnior, 112

São Paulo/SP

Cep: $01221-020$

\section{RESUMO}

OвJetivo. 0 presente estudo teve por objetivo principal avaliar se o bloqueio do fluxo sangüíneo aos ovários, proveniente dos vasos uterinos, pode alterar a função gonadal, resultando na diminuição da secreção de estrogênio e do peso do útero. Métodos. Foi criado um modelo experimental no qual 30 ratas adultas e virgens da cepa Wistar foram incluídas. Após pesagem, os animais foram separados em dois grupos: 0 grupo ligadas, com 20 animais submetidos a laparotomia e ligadura dos ramos ováricos dos vasos uterinos, e o grupo controle, com dez ratas nas quais praticou-se apenas a laparotomia. Após 16 semanas, as ratas foram pesadas e submetidas à eutanásia, tendo seus úteros dissecados e pesados. Calculou-se o quociente entre o peso do útero e o peso do animal.

REsultados. Significância estatíistica não foi observada quando as médias dos pesos uterinos e dos índices entre o peso uterino e o peso dos animais foram comparadas entre os grupos.

Conclusão. A interrupção da irrigação dos ovários, proveniente dos ramos ováricos dos vasos uterinos, não produziu alteração no peso dos úteros dos animais estudados.

UnITERMOS: Ligadura tubária. Efeitos adversos. Distúrbios menstruais.

\section{INTRODUÇão}

A ligadura tubária é o método anticoncepcional mais popular no mundo ${ }^{1,2}$. No Brasil, 31,1\% das mulheres com idade entre 15 e 54 anos estavam esterilizadas. Este número cresceu para 40,1\% após dez anos, caracterizando-a como o método contraceptivo mais usado no País ${ }^{3}$. Em São Paulo, a esterilização feminina não sofreu tal incremento e tem se mantido num patamar de pouco mais de 30\%². Ao lado da grande eficácia, a laqueadura tubária pode provocar efeitos colaterais, destacando-se as anormalidades do fluxo menstrual, dores, alterações no comportamento sexual e emocional ${ }^{2,5}$, constituindo a chamada síndrome pós-ligadura. No entanto, a maioria dos estudos que avaliaram a evolução clínica de mulheres submetidas à ligadura tubária não evidenciou qualquer tipo de distúrbio menstrual ou apenas discretas alterações ${ }^{6-8}$.

Os distúrbios menstruais foram descritos pela primeira vez por Willians et al.. ; neste estudo, os autores compararam 200 mulheres submetidas a ligadura tubária com 5216 pacientes não submetidas ao procedimento (1994 ginecológicas e 3222 obstétricas), durante um período de um a dez anos, e constataram que 19\% das pacientes ginecológicas, 5\% das obstétricas e 24\% das esterilizadas apresentaram distúrbios menstruais, definidos como maior prevalência de sangramento intermenstrual e aumento no fluxo menstrual.

Posteriormente, Muldoon ${ }^{5}$ observou que 374 mulheres esterilizadas, ou 43\% delas, necessitaram de tratamento ginecológico complementar, 19\% de histerectomia e $6 \%$ de outras cirurgias ginecológicas. Diferentemente, Rulin et al. ${ }^{7}$ constataram que importante parcela dos distúrbios menstruais foi referida previamente à ligadura, principalmente em mulheres usuárias de contraceptivos orais combinados.
Outra controvérsia relacionou-se ao tempo de aparecimento dos sintomas; assim, Kassonde \& Bonnarlo não constataram alterações menstruais até 12 meses após a ligadura, enquanto outros estudos ${ }^{11,12}$ observaram-nas após dois anos da cirurgia. Ademais, o risco de internação hospitalar decorrente do sangramento menstrual excessivo foi duas vezes maior em mulheres submetidas a laqueadura do que nas que não foram submetidas ao procedimento ${ }^{13}$. Shobeiri e Atashkhoilit sugerem que as irregularidades menstruais após ligadura tubária são mais freqüentes em mulheres jovens e que tais distúrbios são mais freqüentes durante o período de 12 meses após o procedimento, fortemente relacionados a fatores psicológicos.

Várias hipóteses tentam justificar os mecanismos envolvidos na gênese das alterações menstruais; duas são comumente relatadas: aumento da pressão na microcirculação intra-ovárica ${ }^{15,16}$ e lesão vascular. Na lesão vascular, a hipótese mais aceita, supõe-se que ocorre comprometimento dos ramos ováricos da artéria uterina, que, ao gerar insuficiente irrigação dos ovários, pode desencadear perturbações sobre a esteroidogênese gonadal|3,17.

Além dos aspectos controversos referidos, há uma evidente escassez de estudos na literatura pertinente às alterações menstruais após ligadura tubária. Tais fatos nos motivaram a estudar esta associação em animais de experimentação. Para tanto, elaborou-se um modelo experimental com ratas, pois a vascularização genital desses animais assemelha-se à das mulheres ${ }^{18}$.

Dessa forma, o presente estudo teve por objetivo principal avaliar se o bloqueio do fluxo sangüíneo aos ovários, proveniente dos vasos uterinos, poderia alterar a função gonadal, resultando diretamente na diminuição da secreção de estrogênio e indiretamente na redução do peso do útero ${ }^{19,20}$. 


\section{Métodos}

O estudo foi aprovado pelo Comitê de Ética em Experimentação Animal da Irmandade da Santa Casa de Misericórdia de São Paulo. Foram estudadas 30 ratas adultas virgens, da cepa Wistar, provenientes do Biotério do Departamento de Fisiologia e Biofísica do Instituto de Ciências Biomédicas da Universidade de São Paulo. Após pesadas, as ratas foram divididas aleatoriamente em dois grupos (na proporção de dois animais submetidos a cirurgia para cada animal não submetido a cirurgia), a saber: grupo $A$, denominado ligadas, constituído por 20 animais, e o grupo B, denominado controle, composto por dez, não submetidas a ligadura tubária.

Depois de anestesiados, todos os animais foram submetidos à cirurgia. No grupo controle, realizou-se laparotomia mediana de aproximadamente $3 \mathrm{~cm}$ de extensão, interessando pele, subcutâneo, aponeurose e peritônio parietal. Imediatamente após, procedeu-se o fechamento da incisão, em dois planos, com pontos contínuos de algodão n⿳0 0 (o plano profundo englobou o peritônio parietal e aponeurose e o superficial, exclusivamente a pele). No grupo ligadas, as ratas foram também submetidas a laparotomia com a mesma técnica adotada no grupo controle; entretanto, diferentemente daquele, procedeu-se a abertura da cavidade peritoneal expondo os genitais internos, com identificação dos vasos uterinos e os ramos ováricos. Após cuidadosa dissecção desses ramos, fez-se a ligadura deles próxima ao corno uterino, com fio de poliamida monofilamentar, número 5-0. Em seguida, fez-se o fechamento da incisão abdominal, seguindo-se a mesma técnica utilizada no grupo controle.

Após a recuperação total da anestesia, todos os animais de ambos os grupos foram colocados em gaiolas, com até cinco animais, em regime de água ad libitum e ração balanceada; permaneceram assim durante quatro meses. Nenhum óbito ocorreu durante todo o tempo do experimento.

Transcorrido este período, os animais foram colocados em jejum de 12 horas, pesados e submetidos à eutanásia, na fase de diestro (fase mais longa do ciclo estral, com 60 horas de duração) ${ }^{2 !}$. O diestro foi determinado por meio da leitura de esfregaços vaginais colhidos diariamente com swabs embebidos em soro fisiológico; as lâminas foram coradas com uma gota de solução aquosa de azulde-metileno a 2\% e, após aplicação da lamínula, o esfregaço foi avaliado ao microscópio óptico comum.

Após a extração, os úteros foram dissecados e submetidos à expressão digital para eliminação de seu conteúdo intracavitário. Depois disso, tanto úteros como animais foram pesados em balança eletrônica. Na seqüência, calculou-se o quociente entre o peso do útero e o peso do animal, denominado de índice entre os pesos (IEP), considerado um marcador de atividade estrogênica ${ }^{18}$.

Para análise estatística dos resultados, utilizou-se o teste $t$ de Student para comparar o peso dos animais (antes da cirurgia e antes do sacrifício), o peso do útero e o IEP; o teste t pareado foi aplicado para verificar o ganho ponderal dos animais no período de 16 semanas após a cirurgia. $O$ valor de significância estabelecido foi de $5 \%$.

\section{Resultados}

Do total de animais estudados, um óbito foi registrado no grupo controle. Ao final do experimento, dados obtidos em 29 animais foram analisados. A análise não revelou diferenças significantes entre o peso médio em gramas ( \pm desvio padrão) dos animais dos dois grupos avaliados (A: 221,2 $\pm 19,1$ e $B: 212,6 \pm 22,2$; $p=0,326)$. No transcorrer das 16 semanas, quando os animais permaneceram nas gaiolas, sem nenhuma intervenção ou análise, o ganho ponderal ocorreu nos animais dos dois grupos (respectivamente, $17,9 \pm 18,5$ versus $20,5 \pm 7,1$, grupos $A$ e $B$, com $\mathrm{p}<0,00 \mathrm{I}$ ).

No dia de extração dos úteros, apesar do ganho ponderal, o peso dos animais também não apresentou diferença significante entre os grupos $(240,5 \pm 22,1$ e $233,1 \pm 28,3$; grupos A e B, $\mathrm{p}<0,00 \mathrm{I})$. A Tabela I mostra a análise descritiva dos grupos estudados, em relação ao peso dos animais antes e depois do procedimento.

Em relação ao peso do úteros dissecados dos animais não foram observadas diferenças nos grupos $(0,582 \pm 0,10$ e $0,659 \pm 0,15$, respectivamente, grupos $A$ e $B, p=0,208$; Gráfico I). Não se constataram diferenças nos índices médios ( \pm desvio padrão) obtidos após a correlação entre o peso dos úteros e o peso dos animais depois da eutanásia $(0,002427 \pm 0,00044$ e $0,002850 \pm 0,0007 \mid 4$; respectivamente, grupos $\mid$ e $2, p=0132$, Gráfico 2).

\section{Discussão}

A literatura é controversa no tocante às alterações menstruais após a ligadura tubária; entre os principais, os autores que não aceitam uma maior prevalência destes distúrbios questionam a metodologia dos estudos apresentados, pois não consideram o método anticoncepcional utilizado previamente ${ }^{22}$, nem tampouco a presença de alterações menstruais pré-existentes ${ }^{23}$; nem uma terminologia precisa para definir disfunção menstrual ${ }^{24}$, nem a apreciação de outras possíveis causas de distúrbios menstruais ${ }^{25}$, a ausência de grupos controle e de metodologia cientificamente correta $^{24}$, o número insuficiente de $\operatorname{casos}^{25}$, a falta de um método preciso e objetivo para avaliar a perda sangüínea ${ }^{10,28}$, a diversidade de técnicas empregadas na ligadura ${ }^{7,28}$ e, principalmente, a ausência de estudos prospectivos e randomizados ${ }^{30}$.

\begin{tabular}{lccccc}
\hline Tabela I - Análise descritiva dos grupos estudados, em relação ao peso \\
dos animais antes e depois do procedimento
\end{tabular}




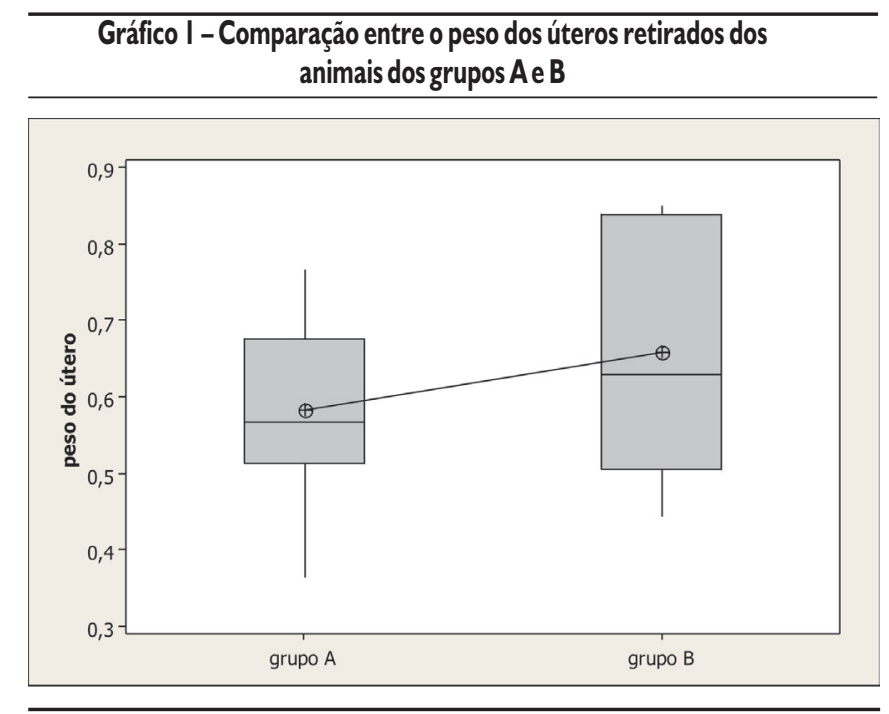

Grupo A: ligadas; grupo B: controle; $\oplus$ média do peso do útero dos animais de cada grupo estudado em gramas

Gráfico 2 - Comparação entre o IEP obtido em animais dos grupos A e B

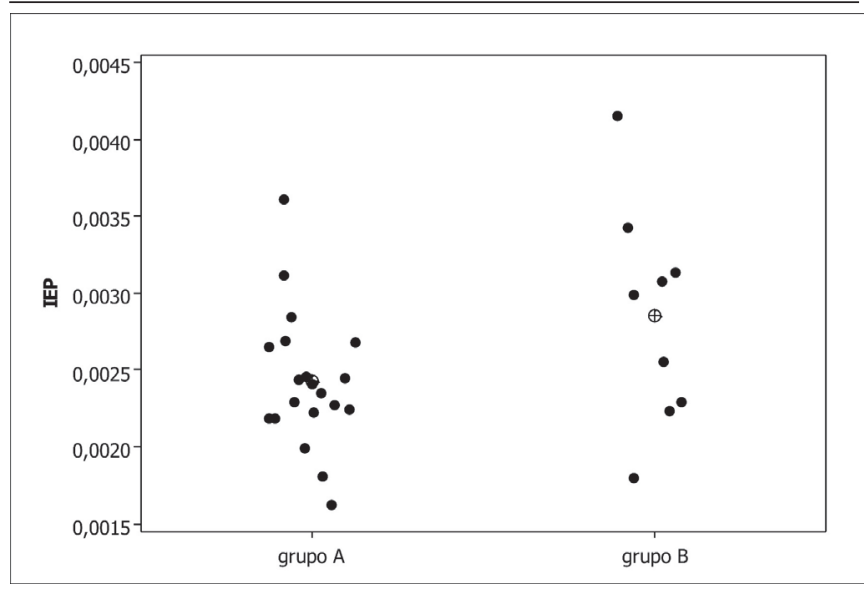

Grupo A: ligadas; grupo B: controle; · IEP de cada animal incluído nos diferentes grupos; $\oplus$ média do IEP dos animais de cada grupo estudado

Em estudo recente realizado por Dede e cols. ${ }^{31}$ demonstrou que, após seguimento das pacientes por três meses com exames ultra-sonográficos com Doppler colorido, parece não haver alterações no fluxo das artérias ovarianas e uterinas após esterilização tubária realizada pela técnica de Pomeroy.

$\mathrm{Na}$ tentativa de explicar a ocorrência destes distúrbios com base nas concentrações séricas de estradiol e progesterona, muitos estudos não obtiveram resultados conclusivos ${ }^{17,32,33}$. Assim, Alvarez et al. ${ }^{34}$ mostraram aumento significativo das concentrações séricas da progesterona após a ligadura; entretanto, os próprios autores criticaram esse achado, face ao curto intervalo de tempo estudado, que foi apenas de seis meses. Por outro lado, Garza-Flores et al. ${ }^{35}$, após um ano de acompanhamento, não evidenciaram qualquer alteração nas concentrações séricas de estrogênio e progesterona.
Thranov et al. ${ }^{36}$, por sua vez, relataram apenas diminuição da progesterona sérica, apesar da presença da ovulação. Fagundes et al. ${ }^{8}$, da mesma maneira, não notaram quaisquer modificações referentes ao intervalo intermenstrual e a duração do sangramento seis meses pós ligadura tubária, apesar da diminuição da progesterona durante a fase lútea sem interferência na resposta endometrial.

No entanto, a redução na incidência do câncer de ovário ${ }^{37}$ e de mama ${ }^{38}$ descrita em mulheres submetidas à esterilização tubárica, constatada em estudos epidemiológicos, renovou o interesse da hipótese da redução da produção de esteróides gerada em mulheres submetidas a laqueadura.

Face a estas discrepâncias e na procura de uma metodologia objetiva e controlada, um modelo experimental com o intuito de contribuir para o esclarecimento deste fenômeno foi elaborado neste estudo.

Nosso principal objetivo foi avaliar se o bloqueio do fluxo sangüíneo para o ovário, decorrente da ligadura do ramo ovárico dos vasos uterinos, poderia resultar numa insuficiência gonadal com conseqüente queda da produção estrogênica, avaliada indiretamente pelo índice obtido entre o peso do útero e a massa corpórea do animal; acreditando que o estrogênio exerce uma ação uterotrófica com efeito indireto no peso do útero ${ }^{19,20,39}$.

Ao se comparar o peso do útero das ratas do grupo ligadas com - controle, apesar de ter sido menor no primeiro grupo, os resultados não mostraram diferenças significativas. Da mesma forma, o IEP (índice entre pesos) mostrou-se inesperadamente semelhante em ambos os grupos, pois supúnhamos que o grupo ligadas exibiria menor peso uterino.

Esses resultados sugerem que a função hormonal do ovário das ratas talvez dependa exclusivamente do fluxo sangüíneo proveniente dos ramos ováricos dos vasos uterinos. Ao explicar a tendência numérica para menor peso uterino, apesar de não ser significativo, no grupo ligadas, valemo-nos do estudo de Borell \& Fernströn ${ }^{40}$, que, ao analisar 300 arteriografias pélvicas de mulheres, verificou grande variabilidade na irrigação dos ovários; assim, havia casos em que a irrigação dos ovários e trompas derivava da artéria ovariana, e outros em que a irrigação dependia exclusivamente de ramos da artéria uterina.

Estas variações podem justificar nossos resultados e a diversidade de manifestações clínicas exibidas pelas mulheres submetidas à ligadura tubária, desde a ausência de alterações menstruais até queixas de metrorragia grave.

Cattanah ${ }^{15}$ especulou que as mudanças na produção hormonal de ovários em mulheres laqueadas não são causadas por irrigação deficiente, mas sim por lesão da microcirculação nos capilares ováricos, resultado do aumento da pressão hidrostática intraluminal observada após a interrupção do fluxo do ramo ovárico da artéria e veia uterinas. Dessa forma, toda a pressão hidrostática proveniente da aorta ou artéria renal seria transmitida aos capilares ováricos, sem atenuação dos vasos uterinos. Essa teoria pode ser extrapolada para o nosso estudo, pois, apesar do suprimento sangüíneo das ratas, oriundo da artéria ovárica, ser intenso, as alterações na fisiologia ovariana poderiam igualmente estar presentes. 
Merece ser enfatizado que nossa metodologia promoveu interrupção de todas as anastomoses entre os vasos uterinos e ováricos, diferentemente dos trabalhos acima comentados, em que a interrupção ocorreu fortuitamente, por ocasião de uma ligadura tubária.

Ainda em relação à metodologia por nós empregada, cabe lembrar que vários autores também utilizaram o mesmo parâmetro (variação do peso do útero) como indicador de atividade estrogênica. Rubin et al. ${ }^{19}$ analisaram como índice de potência estrogênica o quociente entre o peso do útero e o peso total de camundongos; concluíram que representa parâmetro sensível, preciso e conveniente, além de pouco oneroso. No entanto, dois estudos $^{4,42}$ criticaram o uso deste índice, observando que, da mesma maneira que os estrogênios, a progesterona também apresenta a propriedade de estimular o crescimento do útero, fato que poderia tornar este teste menos específico.

Entretanto, estas críticas não contradizem nossa metodologia, pois o nosso propósito básico foi avaliar se teria ocorrido variação na produção hormonal dos esteróides ováricos, decorrente da interrupção do suprimento sangüíneo dos ramos ováricos dos vasos uterinos.

Apesar da laqueadura tubária ser muito prevalente, foi interessante e intrigante constatar uma nítida escassez de estudos pertinentes ao impacto da ligadura tubária sobre a função gonadal, tanto em humanos como em animais. Por isso, o presente estudo, apoiado em metodologia simples e objetiva, possibilitou novas investigações na tentativa de esclarecer as controvérsias ainda existentes.

\section{Conclusão}

Ratas submetidas à ligadura dos ramos ováricos dos vasos uterinos, quando comparadas ao grupo controle não apresentaram qualquer alteração no peso de seus úteros com normal desenvolvimento do peso corpóreo.

\section{Conflito de interesse: não há.}

\section{SUMMARY}

WEIGHT OF UTERUS AFTER LIGATION OF OVARIAN BRANCHES OF THE UTERINE VESSEL

OBIECTIVE. This study aims to evaluate whether the blockage of ovarian irrigation from uterine vessels can change the gonadal function resulting in diminished estrogen secretion and weight of uterus.

METHODS. An experimental model was created including 30 virgin female Wistar rats. After weight determination, animals were separated into two groups: the ligation group with 20 animals, submitted to laparotomy and ligation of the ovarian branches of the uterine blood vessels, and the control group with 10 animals submitted only to laparotomy. Sixteen weeks later the rats were weighed and euthanized, and their uteri dissected and weighed. The index between animal and uteri weights was calculated.

RESULTS. Statistical significance was not observed when the mean uterus weights and the indexes between weight of the uterus and weight of the animals were compared in the two groups.

CONCLUSION. The blockage of ovary vascularization from ovary branches of the uterine vessel did not cause uterine weight alterations. [Rev Assoc Med Bras 2007; 53(2): 166-70]

KEY WORDS: Tubal Sterilization. Adverse effects. Menstruation disturbances/disorders.

\section{REFERÊNCIAS}

I. Church CA, Geller JS. Voluntary female sterilization: number one and growing. Popul Rep C. 1990;10:1-23.

2. Gentile GP, Kaufman SC, Helbig DW. Is there any evidence for a posttubal sterilization syndrome? Fertil Steril. 1998;69:179-86.

3. Berquó E. Ainda a questão da esterilização feminina no Brasil. In: Giffin K, Costa SH, organizadores. Questões da saúde reprodutiva. Rio de Janeiro: Fiocruz; 1999. p.II3-26.

4. Vieira EM, Babiani R, Fabbro AL, Rodrigues Junior AL. Características do uso de anticoncepcionais no Estado de São Paulo. Rev Saúde Pública. 2001;36:263-70

5. Muldoon MJ. Gynaecological illness after sterilization. BMJ. 1972; I:84-9

6. Lu T, Chun D. A long term follow-up study of I.055 cases of postpartum tubal ligation. J Obstet Gynaecol Br Commonw. 1967;74:875-80.

7. Rulin MC, Davidson AR, Philliber SG, Graves WI, Cushman LF. Longterm effect of tubal sterilization on menstrual indices and pelvic pain. Obstet Gynecol. 1993;82:1|8-21.

8. Fagundes ML, Mendes MC, Patta MC, Moura MD, Campos AD, Duartes G, et al. Endometrial status in women submitted to tubal ligation. Rev Bras Ginecol Obstet. 2005;27:529-33.

9. Willians EL, Jones HE, Merril RE. The subsequent course of patients sterilized by tubal ligation. Am J Obstet Gynecol. 1951;61:423-6.

I 0 . Kassonde JM, Bonnar J. Effect of sterilization on menstrual blood loss. British Journal of Obstet Gynecol. 1976;83:572-5.

I I. De Stefano F, Perlman JÁ, Peterson HB, Diamond EL. Long-term risk of menstrual disturbances after tubal sterilization. Am J Obstet Gynecol. | 985; | 52:835-4|.

12. Shain RN, Miller WB, Mitchell GW, Holden AEC, Rosenthal M. Menstrual pattern change I year after sterilization: results of a controlled prospective study. Fertil Steril. 1989;52:193-203.

I 3. Shy KK, Stergachis A, Grothans LG, Wagner EH, Hicht J, Anderson G. Tubal sterilization and risk of subsequent hospital admission for menstrual disorders. Am J Obstet Gynecol. 1992; 166:1698-705.

I 4. Shobeiri MJ, Atashkhoii S. The risk of menstrual abnormalities after tubal sterilization: a case control study. BMC Women's Health. 2005;5:15.

15. Cattanah J. Oestrogen deficiency after tubal ligation. Lancet. 1988; 1 3:847-9.

16. Cattanah JF, Milne BJ. Post-tubal sterilization problems correlated with ovarian steroidogenesis. Contraception. 1988;38:54I-50.

17. Radwanska E, Headley S, Dmowiski P. Evaluation of ovarian function after tubal sterilization. J Reprod Med. 1982;27:376-84.

18. Greene EC. Circulatory system. Anatomy of the rat. New York: Hafner; 1935. p. 1955.

19. Rubin BL, Dorfman AS, Black L, Dorfman RI. Bioassay of estrogens using the mouse uterine response. Endocrinology. 1951;49:429-39.

20. Lan NC, Katzenellenbogen BS. Temporal relationships between hormone receptor binding and biological responses in the uterus. Studies with short and long-acting derivatives of estriol. Endocrinology. 1976;98:220-7.

21 . Astwood EB. Changes in the weight and water content of the normal adult rat. Am J Physiol. 1939; |26:62-70.

22. Lieberman BA, Belsey E, Gordon AG, Wright CSW, Lethworth AT, Noble AD, et al. Menstrual patterns after laparoscopic sterilization using a spring-loaded clip. Br J Obstet Gynecol. 1978;85:376-80. 
Prado RAA ET AL.

23. Edgerton WD. Late complications of laparoscopic sterilization. J Reprod Med. 1977; | 8:275-7.

24. Rioux JE. Late complications of female sterilization: a review of the literature and a proposal for further research. J Reprod Med. 1977; 1 9:329-40.

25. Whitelaw RG. I0 year surbey of 485 sterilizations. BMI. 1 979; 1:32-5

26. Corson SL, Levinson CJ, Batzer FR, Otis C. Hormonal levels following sterilization and hysterectomy. I Reprod Med. 1981;26:363-70.

27. Vessey M, Huggins G, Lawless M, McPherson K, Yeates D. Tubal sterilization: findings in a large prospective study. $\mathrm{Br} J$ Obstet Gynecol. 1 983;90:203-9.

28. Huggins GR, Sondheimer SJ. Complications of female sterilization: immediate and delayed. Fertil Steril. 1984:41:337-55.

29. Rubinstein LM, Lebherz TB, Kleinkopf V. Laparoscopic tubal sterilization: long-term follow-up. Contraception. 1976;13:631-8.

30. Rojansky N, Halbreich U. Prevalence and severity of premenstrual changes after tubal sterilization. J Reprod Med. I99|;36:55।-55.

31 . Dede FS, Akyuz O, Dilbaz B, Caliskan E, Haberal A. Color Doppler flow analysis of uterine and ovarian arteries before and after tubal sterilization: eletrocautery versus pomeroy. Gynecol Obstet Invest. 2006;61:48-9.

32. Alvarez-Sanches F, Segal SJ, Brache V, Adejuwon CP, Faundes A. Pituitary-ovarian function after tubal ligation. Fertil Steril. 1981;36:606-9

33. Donnez J. Wauterz M, Thomas K. Luteal function after tubal sterilization. Obstet Gynecol. 1981;57:65-8.

34. Alvarez F, Faundes A, Brache V. Tejada AS, Segal S. Prospective study of the pituitary-ovarian function after tubal sterilization by the Pomeroy or Uchida techniques. Fertil Steril. 1989;51:604-8.
35. Garza-Flores J, Vazquez-Estrada L, Reyes A, Valero A, Morales Del Olmo A, Alba VM, et al.. Assessment of luteal function after surgical tubal sterilization. Adv Contracept. 1991;7:37|-7.

36. Thranov I, Hertz JB, Kjer JJ, Andersen A, Micic S, Nielsen J, et al. Hormonal and menstrual changes after laparoscopic sterilization by Falope-rings or Filshie-clips. Fertil Steril. 1992;57:75।-5.

37. Miracle-McMahill H, Calle E, Kosinsky A. Tubal ligation and fatal ovarian cancer in a large prospective cohort study. Am J Epidemiol. | 997; | 45:349-57.

38. Calle E, Rodriguez C, Walker KA. Tubal sterilization and risk of breast cancer mortality in US women. Cancer Causes Control. $2001 ; 12: 127-35$

39. Chihal HJW, Peppler RD, Dickey RP. Estrogen potency or oral contraceptive pills. Am J Obstet Gynecol. 1975;121:75-83.

40. Borell V, Fernström I. The adnexal branches of the uterine artery. Acta Radiol. | 1953;40:56।-82.

4I. Jones RJ, Edgren RA. The effects of various steroids on the vaginal histology in the rat. Fertil Steril. 1973;24:28429I.

42. Edgren RA, Sturtevant FM. Potencies of oral contraceptives. Am J Obstet Gynecol. 1976;125:1029-38.
Artigo recebido: I//07/06 Aceito para publicação: 25/I I/06 